\title{
Infinite dimensions and the creation of Universe
}

\author{
Aman Mishra \\ University of petroleum and energy studies, Department of Aerospace Engineering, Dehradun 248007, \\ Uttrakhand, India
}

\begin{abstract}
Science and technology has unraveled many mysteries of our universe but the biggest question of all times still remains an enigma that how our universe came to an existence and what was there before the existence of our universe. A correct explanation will not only let us to understand our birth but will also provide insight to intriguing questions like will our universe have an end and if not how it is going to be. This might give a new outlook to seeing our universe and provide us with the theory of everything. .This paper talks about what was before the big bang, how extra dimensions can be seen, where cosmic censorship can be violated, the constraint energy of the universe, the negative energy and its role in our universe.
\end{abstract}

Keywords: negative energy, naked singularity, extra dimensions, particle decay, particle accelerator, large hadron collider ( $L H C)$

PACS numbers: 98.80.-k; 05.70.-a; 25.75.Gz; 25.75.Dw; 02.40.Xx; 04.20.-q

\section{Introduction}

Before Hubble proved experimentally using Einstein's general theory of relativity that galaxies move away from each other and from us it was believed that the universe was steady and was not expanding, it did not had a beginning nor it will have an end but this discovery thrashed that theory and it is a known fact that our universe is expanding and began with a gigantic explosion known as the big bang but this also did not answer what was before the big bang and why did the universe explode. Now a day's physicists all around the world believe in the latest theory known as the superstring theory, it predicts what happened before the big bang. According to superstrings the universe originally existed in ten dimensions, however the universe was unstable in ten dimensions, it cracked into two pieces, with a small, four dimensional universe peeling off from the rest of the universe, thus forming a sister universe but according to this theory that has shrunk to such a small size (about 100 billion billion times smaller than the nucleus. The superstring theory, therefore, explains the big Bang as a byproduct of a violent transition, the cracking of the ten dimensional universe into two pieces. This paper tries to put in front about the origin of universe and what was there before the big bang but in a different manner than Superstrings. There was absolute nothing before our existence no space, no time absolutely nothing. This absolute nothing comprised of infinite dimensions in a balanced order and everything came into existence from this absolute nothing, so the absolute nothing in one way is everything that is present in our universe. The breakdown of this balanced order system created probably led to the big bang and created everything all planets, stars our space dimensions and even time.

II. How Infinite Dimensions Can Exist In A Balanced Order And Still Be Absolute Nothing.

Dimensions and energy are equivalent. Dimensions are nothing but energy levels, every dimension has an energy level and these energy levels remained in a balanced order and constituted absolute nothing. The sum of all these infinite energy levels was absolute nothing. Absolute nothing would have consisted of positive energy dimensions and their counterparts i.e. negative energy dimension levels.

\section{Formation Of Universe And The Laws Of Universe}

When the break down happened all dimensions which were in balanced order then existed independently. As all dimensions are of different energy level so during the formation of our 3 dimensional universe, all the 3 dimensions came to same level of energy and all the extra energy went into the formation of space and all the three dimensions formed our universe since all these dimensions have a specified energy level so when all the 3 dimensions of our universe came into same energy level it created a energy constraint on our universe. The release of this extra energy at the time of formation might be responsible for the exponential growth of the universe at the time of formation. An energy constraint universe, so a particle cannot cross beyond a certain velocity i.e. the speed of light and solving the equation of Einstein relativistic energy of a particle we can find out the energy constraint of our universe. This hypothesis goes in hand with Einstein's constraint on velocity; this constraint will lead to us to the constraint on energy of our universe. Many dimensions of different energy levels still exists may be some without the formation of space or may be all of them without space or even 
all of them exists with the formation of space whatever be the scenario the constraint on each dimension has blinded us to witness the existence of each other.

\section{Equations}

$$
0=\{- \text { infinity } \ldots . .-3+-2+-1+1+2+3 \ldots \ldots \ldots+\text { infinity }\}
$$

\section{Equation 1.}

It represents the sum of all dimensions which is equivalent to absolute nothing prior to the formation of our universe.

$$
\begin{aligned}
& E=\frac{m c^{2}}{\sqrt{1-v^{2} / c^{2}}} \\
& \lim \frac{m c^{2}}{\sqrt{1-v^{2} / c^{2}}} \\
& m \rightarrow 0 \\
& v \rightarrow c \\
& \lim \frac{c^{2}}{1 / 2\left(1-v^{2} / c^{2}\right) \times-2 v / c^{2}} \\
& v \rightarrow c \\
& \lim -\frac{c^{4}}{v} \times\left(1-v^{2} / c^{2}\right) \\
& v \rightarrow c \\
& \lim -\frac{c^{4}}{v} \times\left(1-\frac{1}{2} \times \frac{v^{2}}{c^{2}}-\frac{1}{8} \times \frac{v^{4}}{c^{4}} \ldots \ldots . .\right. \\
& v \rightarrow c \\
& E=-0.375 c^{3}
\end{aligned}
$$

Equation 2.

It represents the energy constraint of our universe; if somehow the object's mass approaches zero and at the same time while object's mass is approaching zero, the velocity is also equal to the speed of light then we can see the energy constraint of the universe.

$d s^{2}=-\left(1-\frac{2 M}{r}+\frac{Q}{r^{2}}\right) d t^{2}+\frac{d r^{2}}{1-\frac{2 M}{r}+\frac{Q}{r^{2}}}+r^{2} d \Omega^{2}$ Einstein and Rosen made a complete ad hoc

mutilation to the theory-they found that they had to reverse the sign of the electromagnetic stress energy tensor, so the mutilated geometry in terms of Schwarzschild coordinates is

$$
d s^{2}=-\left(1-\frac{2 M}{r}-\frac{\varepsilon^{2}}{r^{2}}\right) d t^{2}+\frac{d r^{2}}{1-\frac{2 M}{r}-\frac{\varepsilon^{2}}{r^{2}}}+r^{2} d \Omega^{2}
$$

Taking $\mathrm{M}=0$ the metric reduces to

$$
d s^{2}=-\left(1-\frac{\varepsilon^{2}}{r^{2}}\right) d t^{2}+\frac{d r^{2}}{1-\frac{\varepsilon^{2}}{r^{2}}}+r^{2} d \Omega^{2}
$$


Thus with $\mathrm{M}=0$, the coordinate change $u^{2}=r^{2}-\frac{\varepsilon^{2}}{2}$ results in

$$
d s^{2}=-\frac{u^{2}}{u^{2}+\frac{\varepsilon^{2}}{2}} d t^{2}+d u^{2}+\left(u^{2}+\frac{\varepsilon^{2}}{2}\right)^{2} d \Omega^{2}
$$

\section{Equation 3}

It represents a mass less, quasicharged object whose energy density is everywhere negative.

\section{EQUATIONS EXPLAINED}

Equation 1:- It describes that everything is absolute nothing and how our universe came into existence from absolute nothing to everything that we see and beyond. The absolute nothing comprised of infinite dimensions so arranged that the universe was in a balanced state.

Equation 2:- It describes when a quasi charged particle moving with almost or speed of light then at that instant it decays. Meaning the mass approached to zero (here we are referring to this particle only and not at its decay part) and velocity was almost the speed of light so at that very instant the relativistic energy becomes negative and reaches the constraint energy of the universe

Equation 3:- It describes the space-time metric of a mass less, quasi charged particle whose energy density is everywhere negative, Einstein and Rosen found this geometry and this geometry predicted the formation of a naked singularity.

Combined Equation 2\&3 :- When a quasi charged particle moving with speed of light decays, it creates a negative energy space-time which results in the formation of a naked singularity(thus violating cosmic censorship) and that is the point of constraint energy of our universe or a gateway to a different dimension.

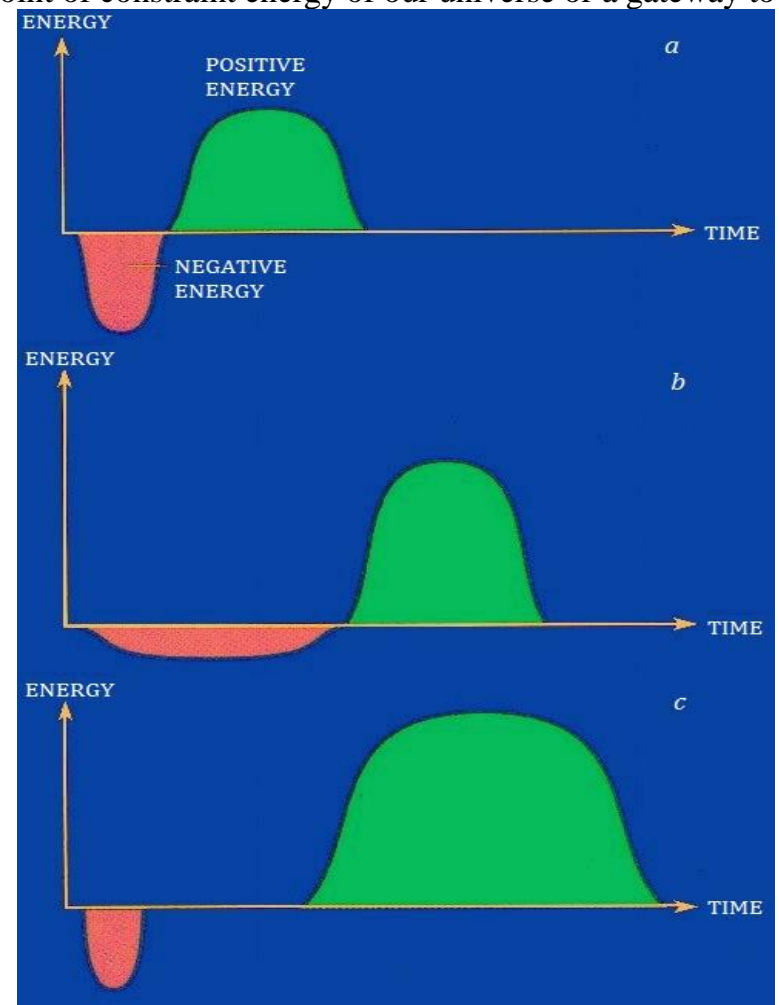

Fig. 1. Represents pulse of negative energy permitted by quantum theory, (a, b) says the longer the pulse lasts the weaker it must be. Secondly, a pulse of positive energy must flow; the magnitude of the positive pulse must exceed that of the initial negative one. (c) Tells us that longer the time interval between the two pulses, the larger the positive one must be- an effect known as quantum interest. 


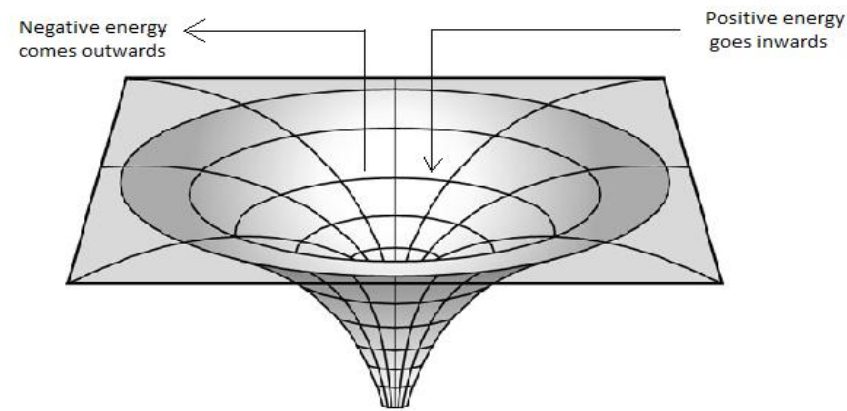

Fig.2. shows the state when the particle reaches the energy constraint, there is negative energy so positive energy must flow inwards but the value of positive energy flowing inwards should be more than the negative energy magnitude.

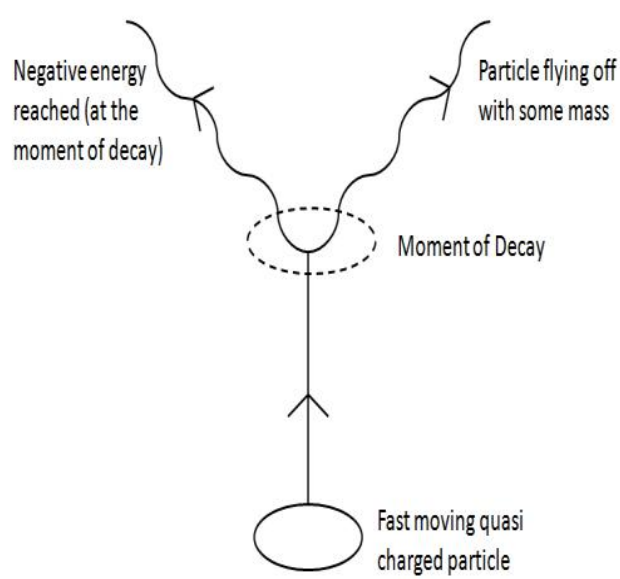

Fig 3. Shows the decay of a quasi charged particle, at the moment of decay the energy density becomes negative and reaches the constraint energy of the universe.

\section{Experiment (Particle Accelerator)}

Take a quasi charged particle accelerate it to almost the speed of light in a particle accelerator(like large hadron collider) and when it decays observe the space-time at that instant as there will be negative relativistic energy observe phenomenon like anti gravity or observe the change in energy at that instant because the metric results in the formation of a naked singularity (not a black hole) and due to the quantum effects and properties of negative energy, there is a flow of positive and negative energy but more positive energy will flow inwards as compared to the negative energy coming out so observe the total change in the energy at that instant.

\section{Conclusions And Discussions}

1. This paper deals with the origin of universe from the state of absolute nothing to everything and this evolution process contained our big bang.

2. There are infinite dimensions and every dimension has its energy constraint. This state's the formation of our universe from a set of infinite dimensions and not merely ten or eleven. This does not talk about a sister universe with whose fission lead to the big bang. Our universe is expanding in hyper dimension which might not necessarily be a space dimension but could be a higher energy level dimension.

3. The calculated energy constraint of our universe comes out to be negative, meaning at the point where the object reaches the energy constraint the space-time curvature becomes extreme. As energy must be conserved the production of negative energy must be accompanied by flow of positive energy inwards.

4. The geometry when the constraint energy is reached is that of the mass less, quasicharged object whose energy density is everywhere negative as calculated by Einstein and Rosen .It gives a naked singularity, the mutilation of the Reisssner-Nordstrom geometry by Einstein and Rosen by maiming of the theory, $Q^{2}=-\varepsilon^{2}$ was done to avoid the presence of a naked singularity.

5. When the constraint energy is reached by the quasicharged particle there will be a naked singularity. 
6. As the amount of positive energy flowing inwards is greater than the negative energy coming outwards it means that there is a net reduction of the energy from our universe at that singular point, or in other words energy gets extracted at that point of the system so entropy decreases at this singular point.

7. Particle decay of a quasi charged particle moving with almost the speed of light results in the formation of a naked singularity, resulting in the observation of a new dimension.

\section{Acknowledgments}

I want to show my gratitude towards Dr. Ugur guven(Aerospace engineer/ Nuclear engineer) whose intellect and guidance has given me strength, knowledge and a proper direction to be able to understand astrophysics and think about various possibilities of this enigmatic universe.

\section{References}

[1] Michio Kaku;Jennifer Trainer Thomson., Beyond Einstein: Superstrigs And Quest For Final Theory, Oxford University Press.

[2] Kip S Thorne; W.W Norton, Black Holes And Time Warps: Einstein's Outrageous Legacy(1994)

[3] Matt Viser, Lorentzian Wormholes: From Einstein To Hawking, American Institute Of Physics Press(1996)

[4] Eric Poisson,A Relativist's Toolkit: The Mathematics Of Black Hole Mechanics

[5] Http://Www.Bibliotecapleyades.Net/Ciencia/Negativeenergy/Negativeenergy.Htm 